\title{
Synthesis of 2,2-Di-C-methyl-2-deoxy- and 4,4-Di-C-methyl- 4-deoxypyranosides via Michael Addition of Conjugated Enopyranosiduloses
}

\author{
Nobuya Kawauchi, Ken-ichi Sato, Juji Yoshimura, and Hironobu Hashimoto* \\ Laboratory of Chemistry of Natural Products, Faculty of Science, Tokyo Institute of \\ Technology, Nagatsuta, Midori-ku, Yokohama 227
}

(Received October 7, 1986)

\begin{abstract}
Three hexopyranosides having gem-di- $C$-methyl group at 2- or 4-positions were synthesized by Michael addition to 2-enopyranosid-4-ulose and 3-enopyranosid-2-ulose derivatives, respectively, followed by hydroboration. A heptopyranosid-6-ulose having gem-di- $C$-methyl group at C-4 was also synthesized from 4enopyranosid-6-ulose derivative in a similar way.
\end{abstract}

In recent years, carbohydrates have been used widely as chiral sources for synthesis of natural products. ${ }^{1)}$ gem-Dialkylation of carbohydrates has progressed on this utilization. For example, the following gemdialkyl groups have been constructed: a) $C$-cyano- $C$ nitromethyl as well as $C$-methyl- $C$-nitromethyl group from the $C$-nitromethylene group by Michael addition, ${ }^{2)}$ b) $C$-(ethoxycarbonylmethyl)- $C$-methyl group by enolate alkylation of the ulose derivative, ${ }^{3)}$ c) $C$ formylmethyl- $C$-vinyl group from $C$-[2-(vinyloxy)ethylidene] group by Claisen rearrangement. ${ }^{4)}$ On the other hand, introduction of gem-dimethyl group into the carbohydrate skeleton has not yet been reported except a recently published paper ${ }^{5)}$ and our preliminary one, ${ }^{6)}$ although gem-dimethylation has been achieved for non-carbohydrate compounds by several methods. ${ }^{7-11)}$ In this paper we should like to report on the synthesis of gem-di-C-methylpyranosides by Michael addition of methylcuprates(I) to conjugated enopyranosiduloses. This method has been proved to be useful for construction of gem-dimethyl function at C-2 and C-4 positions of hexopyranosides, while the method by hydrogenolytic cleavage of spirocyclopropane ring for that at C-3 position. $\left.{ }^{6}\right)$

\section{Results and Discussion}

Four kinds of conjugated enopyranosiduloses 2-5 were used for this study. The first two branched-chain enopyranosiduloses $\mathbf{2}$ and $\mathbf{3}$ were derived from a common starting compound, i.e., methyl 6- $O$-trityl- $\alpha$-Dglycero-2-enopyranosid-4-ulose (6). ${ }^{12)}$ Conjugate 1,4addition of a lithium (cyano)dimethylcuprate(I), $\mathrm{Me}_{2} \mathrm{Cu}$ (CN) $\mathrm{Li}_{2}$, to 6 followed by treatment with chlorotrimethylsilane, gave the corresponding enol trimethylsilyl ether 7. Dehydrosilylation of 7 with $p$ benzoquinone in the presence of palladium(II) acetate $^{13)}$ afforded $\mathbf{2}$ in $55 \%$ yield from 6 . Alternatively, the enone 2 was prepared from methyl 4,6-Obenzylidene-2-deoxy-2- $C$-methyl- $\alpha$-D-altropyranoside (9) via the 3-enopyranoside $\mathbf{8}$, which was formed selectively by treatment of the 3-mesylate 10 of 9 with potassium $t$-butoxide. Palladium-catalyzed enone formation $^{14)}$ was proved to be effective for the enolate 8 to give enone 1 , which was converted into 2 by a conven- tional tritylation. The total yield of $\mathbf{2}$ from $\mathbf{9}$ was $11.4 \%$. The latter route proved to be rather convenient than the previous on ${ }^{15)}$ in the steps of elimination and enone formation.

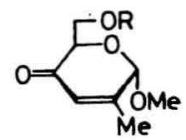

$1 \mathrm{R}=\mathrm{H}$

$2 \mathrm{R}=\mathrm{Tr}$

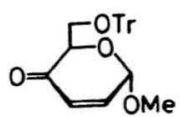

6

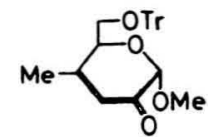

3

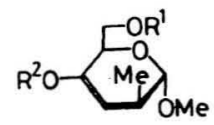

$7 \mathrm{R}^{\mathrm{l}}=\mathrm{Tr}, \mathrm{R}^{2}=\mathrm{TMS}$

$8 \mathrm{R}^{\mathrm{l}}, \mathrm{R}^{2}=\mathrm{PhCH}$

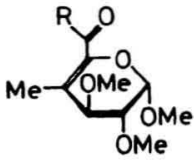

$4 \mathrm{R}=\mathrm{H}$

$5 \mathrm{R}=\mathrm{Me}$

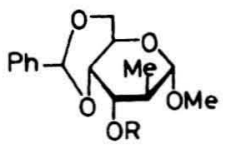

$9 \mathrm{R}=\mathrm{H}$

$10 \mathrm{R}=\mathrm{Ms}$
On the other hand, the enone 3 was already prepared $^{16)}$ by pyridinium chlorochromate oxidation of 1,2-adduct 11 obtained by treatment of 6 with methyllithium. In the addition reaction the ratio of 11 and its 4 -epimer was proved to be $4: 3$, while with methylcerium(III) reagent ${ }^{17)}$ instead of methyllithium 11 was obtained exclusively in a quantitative yield. Thus it is noteworthy that the methylcerium reagent tends to facilitate axial attack of carbanion, which was also observed in the reaction of methyl 3,4-dideoxy-6-Otrityl- $\alpha$-D-glycero-3-enopyranosid-2-ulose. ${ }^{18)} \quad$ Furthermore, oxidation of $\mathbf{1 1}$ with pyridinium fluoroformate $^{19)}$ instead of chloroformate gave 3 in a higher yield (86\%), indicating that this less acidic oxidant are convenient and effective for allylic alcohol.

The enone 4 was derived from methyl 6-O-benzyl2,3-di- $O$-methyl- $\alpha$-D-glucopyranoside $(12)^{20)}$ according to a similar strategy to Hanessian et al. ${ }^{21)}$ Swern oxidation of 12 gave the corresponding 4-ulose, which was converted stereoselectively with methylmagnesium iodide into the 4-C-methyl derivative 13 of $\mathrm{D}$-galacto configuration in $77 \%$ yield in two steps. Methylation of 13 followed by hydrogenolytic removal of 6-Obenzyl group and Swern oxidation gave a dialdose 14 in $79 \%$ yield. Then, 4 was obtained in $65 \%$ yield by treatment of 14 with calcium hydroxide. As shown in 
Table 1 the reaction of 4 with various methylcuprates(I) gave in good yields the 1,2-adduct 15, from which the enone 5 was derived quantitatively by Swern oxidation.

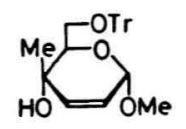

11

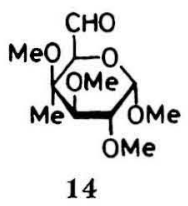

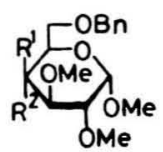

$12 \mathrm{R}^{1}=\mathrm{H}, \mathrm{R}^{2}=\mathrm{OH}$

$13 \mathrm{R}^{1}=\mathrm{OH}, \mathrm{R}^{2}=\mathrm{Me}$

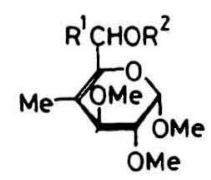

$15 \mathrm{R}^{1}=\mathrm{Me}, \mathrm{R}^{2}=\mathrm{H}$

$16 \mathrm{R}^{1}=\mathrm{Me}, \mathrm{R}^{2}=\mathrm{Ac}$

$17 \mathrm{R}^{1}=\mathrm{R}^{2}=\mathrm{H}$
The reaction of these enones 2,3 , and 5 with lithium (cyano)dimethylcuprate(I), $\mathrm{Me}_{2} \mathrm{Cu}(\mathrm{CN}) \mathrm{Li}_{2}$, gave preferentially 1,4-adducts, gem-di-C-methyl-pyranosiduloses 18, 19, and 20, respectively, in high yields as shown in Table 1 . In the case of 3 very small amount of by-product, the $\beta$-anomer of $\mathbf{1 9}$, was isolated. On the other hand, the reaction of the enal $\mathbf{4}$ afforded an

Table 1. Reaction of Methyl Branched Enones 2-5 with Lithium Methylcuprates (I)

\begin{tabular}{cccc}
\hline \multirow{2}{*}{ Enone } & \multirow{2}{*}{ Product } & \multicolumn{2}{c}{ Yield $/ \%$} \\
\cline { 3 - 4 } & & $\mathrm{Me}_{2} \mathrm{CuLi}$ & $\mathrm{Me}_{2} \mathrm{Cu}(\mathrm{CN}) \mathrm{Li}_{2}$ \\
\hline $\mathbf{2}$ & $\mathbf{1 8}$ & 72 & 77 \\
& 5 -epimer of $\mathbf{1 8}$ & 8.4 & 3.1 \\
$\mathbf{3}$ & $\mathbf{1 9}$ & 82 & 93 \\
& $\beta$-anomer of $\mathbf{1 9}$ & 6.9 & 4.1 \\
$\mathbf{4}^{\text {a) }}$ & $\mathbf{1 5}$ & 62 & 90 \\
& $\mathbf{1 7}$ & 12 & trace \\
$\mathbf{5}$ & $\mathbf{2 0}$ & 83 & 89 \\
\hline
\end{tabular}

a) With $\mathrm{Me}_{5} \mathrm{Cu}_{2} \mathrm{Li}_{3}$ the yield of $\mathbf{1 5}$ was $93 \%$. epimeric mixture of 1,2-adduct 15 together with small amount of reduced product 17 . The distinct difference of regioselectivity in the addition reactions between 4 and 5 coincide with the fact that aldehydes react easily with lithium organocuprates, while ketones not. ${ }^{22)}$

Thus, it was turned out that gem-dimethylation in hexopyranoside ring could be performed very easily using conjugate addition to the enopyranosiduloses. Furthermore, in the cases of 18 and 19 asymmetric carbons lost during the enone formation was also recovered by hydroboration of the silyl enolates ${ }^{23)}$ e.g., $18 \rightarrow \mathbf{2 1} \rightarrow \mathbf{2 4}$ or $\mathbf{1 9} \rightarrow \mathbf{2 3} \rightarrow \mathbf{2 6}$. The enolates 21 and 23 were obtained by quenching the above mentioned Michael addition with chlorotrimethylsilane in good yields. Hydroborations of $\mathbf{2 1}$ and $\mathbf{2 3}$ were examined using various reagents and the products were analyzed after hydrolysis and acetylation. As summarized in Table 2, hydroboration with 3 equivalents of diborane gave the best results. In the case of 21, only 2-deoxy2,2-di- $C$-methyl-hexopyranoside derivative 24 was obtained as hydroboration product, where a small amount of 3-deoxy-D-erythro-hexopyranoside 25 and 3 -enopyranoside 22 were also formed. In the case of 23, 4-deoxy-4,4-di-C-methyl-D-xylo- 26 and D-arabinohexopyranoside derivatives 28 were obtained in a ratio of 3 to 1, and 3-deoxy-D-erythro-hexopyranoside 27 was also formed. Hydroboration with borane formed in situ from sodium borohydride and Lewis acids such as titanium(III) chloride ${ }^{24)}$ and cobalt(II) chloride ${ }^{25)}$ gave also the desired products in moderate yields, although recovery of the uloses were increased. On the other hand, hydroboration with disiamylborane and 9-borabicyclo[3.3.1]nonane (9-BBN) gave small amount of the reduced by-products, 25 and 27 , while the uloses were recovered in high yield. Only in the case of $\mathbf{2 3}$ hydroboration with disiamylborane afforded 26, albeit in $17 \%$ yield, stereoselectively. The structures of $\mathbf{2 4 , 2 6}$, and 28 were confirmed on the basis of two acetyl signals and the coupling constants of methine protons

Table 2. Hydroboration ${ }^{\text {a) }}$ of 21 and 23

\begin{tabular}{|c|c|c|c|c|c|c|c|c|c|}
\hline \multirow{2}{*}{$\frac{\text { Enolate }}{21}$} & \multicolumn{2}{|l|}{ Reagent } & \multicolumn{5}{|c|}{ Product $^{\text {b) }}$ and Yield $/ \%$} & \multicolumn{2}{|c|}{ Recovered ulose $/ \%$} \\
\hline & Borane ( 1.1 equiv) & 24 & Trace & & & & & 18 & 70 \\
\hline 21 & Borane (3.0 equiv) & 24 & 43 & 25 & 6.4 & 22 & 5.8 & & \\
\hline 21 & Borane (9.0 equiv) & 24 & 44 & 25 & 8.6 & 22 & 4.6 & & \\
\hline 21 & $\mathrm{NaBH}_{4}, \mathrm{TiCl}_{3}$ & 24 & 12.5 & 25 & 12.5 & 22 & 10 & 18 & 30 \\
\hline 21 & $\mathrm{NaBH}_{4}, \mathrm{CoCl}_{2}$ & 24 & 26 & 25 & Trace & 22 & 2.0 & 18 & 51 \\
\hline 21 & Disiamylborane & 24 & Trace & 25 & 14 & & & 18 & 76 \\
\hline 21 & $9-\mathrm{BBN}^{\mathrm{c})}$ & 24 & Trace & 25 & 7.8 & & & 18 & 70 \\
\hline 23 & Borane (1.1 equiv) & 26 & 26 & 28 & 5.3 & 27 & 9.0 & & \\
\hline 23 & Borane ( 3.0 equiv) & 26 & 29 & 28 & 9.7 & 27 & 13 & & \\
\hline 23 & Borane (9.0 equiv) & 26 & 22 & 28 & 6.3 & 27 & 13.5 & & \\
\hline 23 & $\mathrm{NaBH}_{4}, \mathrm{CoCl}_{2}$ & 26 & 24 & 28 & 12 & 27 & Trace & & \\
\hline 23 & $\mathrm{NaBH}_{4}, \mathrm{TiCl}_{3}$ & 26 & 27 & 28 & 9.1 & 27 & 16 & & \\
\hline 23 & Disiamylborane & 26 & 17 & & & 27 & 13 & 19 & 43 \\
\hline 23 & $9-\mathrm{BBN}^{\mathrm{c})}$ & 26 & Trace & & & 27 & 7.9 & 19 & 57 \\
\hline
\end{tabular}

a) Followed by treatment with alkaline hydrogen peroxide. b) Isolated after acetylation with acetic anhydride and pyridine. c) 9-Borabicyco[3.3.1]nonane. 


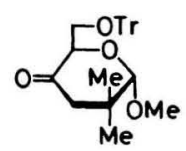

18

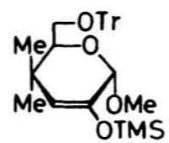

23

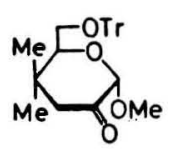

19

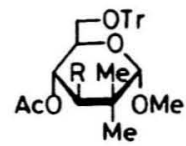

$24 \mathrm{R}=\mathrm{OAc}$

$25 \mathrm{R}=\mathrm{H}$ with acetoxyl groups, i.e., $J_{3,4}=8.0 \mathrm{~Hz}, J_{2,3}=7.8 \mathrm{~Hz}$, and $J_{2,3}=4.0 \mathrm{~Hz}$, respectively. While those of 25 and 27 by one acetyl signal and the coupling constants of methine protons with acetoxyl group, i.e., $J_{3 a x, 4}=7.8$ $\mathrm{Hz}$ and $J_{2,3 \mathrm{ax}}=13.3 \mathrm{~Hz}$, respectively.

The formation of the deoxygenated derivatives, 25 and 27, may be rationalized by trans $\beta$-elimination ${ }^{26)}$ of trimethylsiloxyborane from the hydroboration products, giving the corresponding de(trimethylsilyloxy) derivative. Actually in the reaction of 21 , the de(trimethylsilyloxy) intermediate $\mathbf{2 2}$ was isolated. Further hydroboration of $\mathbf{2 2}$ followed by oxidation may give 25, where the stereoselectivity was well-explained by a half-chair conformation as depicted in the following scheme.

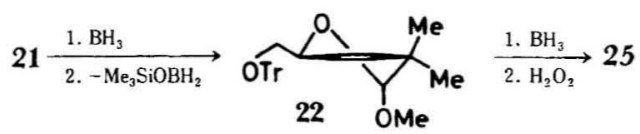

Thus, Michael addition of lithium methylcuprate to 2-enopyranosid-4-ulose and 3-enopyranosid-2-ulose derivatives, followed by hydroboration-oxidation, opened the synthetic routes of hexopyranosides having gem-di-C-methyl group at 2- and 4-positions, respectively.

\section{Experimental}

General Methods. Melting points determined with a Yanagimoto micro melting point apparatus, were uncorrected. Optical rotations were measured in chloroform, by using a 0.5-dm tube with a Carl Zeiss LEP-Al polarimeter. Infrared spectra were recorded on a Hitachi EPI-G2 grating spectrometer. ${ }^{1} \mathrm{H}$ and ${ }^{13} \mathrm{C}$ NMR spectra were recorded at 100 $\mathrm{MHz}$ with a JEOL JMN PS-100 spectrometer and at 22.5 $\mathrm{MHz}$ with a JEOL FX-90Q spectrometer, respectively, in $\mathrm{CDCl}_{3}$ with tetramethylsilane as internal standard. Column chromatography was performed on silica gel (Wakogel C300: Wako Pure Chemical Industries, Ltd.) ${ }^{1} \mathrm{H}$ and ${ }^{13} \mathrm{C}$ NMR data of pyranosides having $\mathrm{gem}$-di-C-methyl group are summarized in Table 3.

Methyl 2,3-Dideoxy-2- $C$-methyl-6- $O$-trityl- $\alpha$-D-glycero-hex2-enopyranosid-4-ulose (2). To a mixture of palladium(II) acetate $(112 \mathrm{mg}, 0.5 \mathrm{mmol}$ ) and $p$-benzoquinone ( $54 \mathrm{mg}, 0.5$ mmol) was added under argon a solution of $7(460 \mathrm{mg}, 0.95$ $\mathrm{mmol}$ ), whose preparation is described below, in acetonitrile
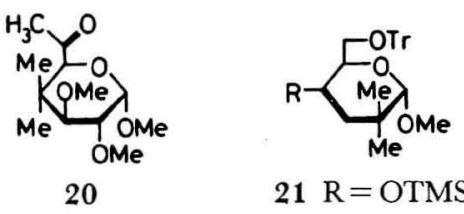

$$
21 \mathrm{R}=\text { OTMS }
$$$$
22 \mathrm{R}=\mathrm{H}
$$

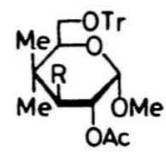

$26 \mathrm{R}=\mathrm{OAc}$

$27 \mathrm{R}=\mathrm{H}$

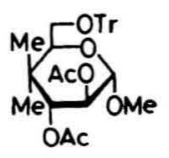

28

$(4 \mathrm{ml})$. The mixture was stirred at $50^{\circ} \mathrm{C}$ for $6 \mathrm{~h}$, and fractionated directly on a silica-gel column with benzene-acetone (15:1) to give 2 (24l mg, 62\%). IR and NMR data of 2 coincided with the reported data. ${ }^{15)}$

Methyl 3,4-Dideoxy-4-C-methyl-6-O-trityl- $\alpha$-D-glycero-hex3-enopyranosid-2-ulose (3). A solution of pyridinium fluorochromate $(2.36 \mathrm{~g}, 11.9 \mathrm{mmol})$ and $11(2.72 \mathrm{~g}, 6.6 \mathrm{mmol})$ in dichloromethane $(84 \mathrm{ml})$ was stirred at room temperature for $6 \mathrm{~h}$. The undissolved materials were filtered off, and the filtrate was washed with $2.5 \%$ aqueous sodium hydroxide and water, and dried with potassium carbonate. Evaporation of the filtrate gave a syrup, which was purified on a short column of silica gel with hexane-ethyl acetate (18:1) to afford $3(2.33 \mathrm{~g}, 86 \%)$ as a colorless syrup. ${ }^{1} \mathrm{H}$ NMR data was identical with those reported. ${ }^{16)}$

Methyl 4-Deoxy-2,3-di- $O$-methyl-4- $\boldsymbol{C}$-methyl- $\beta$-L-threohexodialdo-4-enopyranoside-(1,5) (4). To a solution of 14 $(500 \mathrm{mg}, 2.0 \mathrm{mmol})$ in tetrahydrofuran $(5 \mathrm{ml})$ and water $(5$ $\mathrm{ml}$ ), was added $0.5 \mathrm{M}\left(1 \mathrm{M}=1 \mathrm{~mol} \mathrm{dm}^{-3}\right)$ aqueous solution of barium hydroxide $(4 \mathrm{ml})$. The mixture was kept at room temperature for $6 \mathrm{~h}$, then saturated with ammonium chloride and extracted with chloroform. The extract was washed with aqueous sodium chloride, dried over magnesium sulfate, and evaporated to give a crude mixture of products, which was fractionated on a column of silica gel with hexaneacetone $(5: 1)$ to give 4 (284 mg, 65\%), methyl 2,3-di- $O$ methyl-4- $C$-methylene- $\beta$-L-threo-hexodialdo-4-enopyranoside$(1,5)$ (17 mg, 3.9\%), and starting material (29 mg, 5.8\%).

4: $\mathrm{Mp} 148-150^{\circ} \mathrm{C},[\alpha]_{\mathrm{D}}^{20}+31.2^{\circ}\left(c 0.7, \mathrm{CHCl}_{3}\right)$. IR $(\mathrm{KBr})$ $1680(\mathrm{CO})$ and $1640 \mathrm{~cm}^{-1}(\mathrm{C}=\mathrm{C}),{ }^{1} \mathrm{H}$ NMR $\delta=5.06(1 \mathrm{H}, \mathrm{d}$, $\mathrm{H}-1), 3.92\left(1 \mathrm{H}, \mathrm{dd}, J_{1,2}=2.2 \mathrm{~Hz}, \mathrm{H}-2\right), 3.60\left(1 \mathrm{H}, \mathrm{d}, J_{2,3}=7.8 \mathrm{~Hz}\right.$, $\mathrm{H}-3$ ), 9.76 (1H, s, H-6), 2.12 (3H, s, C-Me), 3.44 (3H, s, OMe) and $3.52(6 \mathrm{H}, \mathrm{s}, \mathrm{OMe})$.

Found: $\mathrm{C}, 55.38 ; \mathrm{H}, 7.48 \%$. Calcd for $\mathrm{C}_{10} \mathrm{H}_{16} \mathrm{O}_{5}: \mathrm{C}, 55.54$; $\mathrm{H}, 7.46 \%$.

The exo-methylene compound was a syrup, and had $[\alpha]_{\mathrm{D}}^{20}$ $+10.1^{\circ}\left(c 0.88, \mathrm{CHCl}_{3}\right) .{ }^{1} \mathrm{H}$ NMR $\delta=4.96\left(1 \mathrm{H}, \mathrm{d}, J_{1,2}=3.0 \mathrm{~Hz}\right.$, $\mathrm{H}-1$ ), 3.6-3.7 (2H, m, H-2 and H-3), 3.98 (1H, bs, H-5), 9.54 (1 H, bs, H-6), $3.52(3 \mathrm{H}, \mathrm{s}, \mathrm{OMe})$, and $3.56(6 \mathrm{H}, \mathrm{s}, \mathrm{OMe})$.

Found: $\mathrm{C}, 55.41: \mathrm{H}, 7.60 \%$. Calcd for $\mathrm{C}_{10} \mathrm{H}_{16} \mathrm{O}_{5}: \mathrm{C}, 55.54$; H, $7.46 \%$.

Methyl 4,7-Dideoxy-4- $C$-methyl-2,3-di- $O$-methyl- $\beta$-L-threohept-4-enopyranosid-6-ulose (5). To a suspension of cerium(III) chloride (318 mg, $1.3 \mathrm{mmol}$ ) in tetrahydrofuran $(5 \mathrm{ml})$, was added an ethereal solution of methyllithium (1.2 M solution, $1.1 \mathrm{ml}, 1.3 \mathrm{mmol}) \mathrm{at}-78^{\circ} \mathrm{C}$, and then after $1 \mathrm{~h}$ a solution of $4(260 \mathrm{mg}, 1.1 \mathrm{mmol})$ in tetrahydrofuran $(5 \mathrm{ml})$. The mixture was kept at $-78^{\circ} \mathrm{C}$ for $4 \mathrm{~h}$ and poured into a saturated ammonium chloride solution and extracted with dich- 
Table 3. ${ }^{1} \mathrm{H}$ and ${ }^{13} \mathrm{C}$ NMR Data of Pyranosides Having gem-Di-C-methyl Group

\begin{tabular}{|c|c|c|c|c|c|c|c|c|c|}
\hline Compound & 1 & 2 & 3 & 4 & 5 & 6 & $\mathrm{C}-\mathrm{Me}$ & $\mathrm{OMe}$ & Others \\
\hline $18\left({ }^{1} \mathrm{H}\right)$ & $4.36 \mathrm{~s}$ & & $\begin{array}{l}2.08 .2 .50 \mathrm{~d} \\
(J=15.4)\end{array}$ & & $\begin{array}{c}4.02 \mathrm{dd} \\
\left(J_{5,6}=\dot{4} .2\right)\end{array}$ & $\begin{array}{c}3.30 \mathrm{dd}, 3.42 \mathrm{t} \\
\left(J_{5,6^{\prime}}=J_{6,6^{\prime}}=8.8\right)\end{array}$ & $\begin{array}{l}0.94 \mathrm{~s} \\
1.18 \mathrm{~s}\end{array}$ & $3.44 \mathrm{~s}$ & $7.1-7.5 \mathrm{~m}(\mathrm{Tr})$ \\
\hline $18\left({ }^{13} \mathrm{C}\right)$ & $103.08 \mathrm{~d}$ & $37.39 \mathrm{~s}$ & $47.24 t$ & $205.51 \mathrm{~s}$ & 73.16 & $61.64 t$ & $\begin{array}{l}25.23 q \\
25.82 q\end{array}$ & $54.57 \mathrm{q}$ & a) \\
\hline $19\left({ }^{1} \mathrm{H}\right)$ & $4.58 \mathrm{~s}$ & & $\begin{array}{l}1.96 \mathrm{dd}(J=1.0), \\
2.64 \mathrm{~d}(J=14.2)\end{array}$ & & $\begin{array}{c}4.16 \mathrm{dd} \\
\left(J_{5,6}=7.4\right)\end{array}$ & $\begin{array}{c}3.20 \mathrm{t}, 3.18 \mathrm{dd} \\
\left(J_{5,6^{\prime}}=J_{6,6^{\prime}}=3.8\right)\end{array}$ & $\begin{array}{l}1.76 \mathrm{bs} \\
1.78 \mathrm{~s}\end{array}$ & $3.60 \mathrm{~s}$ & $7.1-7.6 \mathrm{~m}(\mathrm{Tr})$ \\
\hline $19\left({ }^{13} \mathrm{C}\right)$ & 100.24 & 202.58 & 51.04 & 39.88 & 75.53 & 63.07 & $\begin{array}{l}20.43 \\
26.82\end{array}$ & 54.94 & b) \\
\hline $24\left({ }^{1} \mathrm{H}\right)$ & $5.16 \mathrm{~s}$ & & $\begin{array}{l}5.22 \mathrm{~d} \\
\left(J_{3,4}=J_{4,5}=8.0\right)\end{array}$ & $5.08 \mathrm{t}$ & $3.86 \mathrm{~m}$ & $3.12 \mathrm{dd}, 3.20 \mathrm{t}$ & $\begin{array}{l}0.96 \mathrm{~s} \\
1.12 \mathrm{~s}\end{array}$ & $3.38 \mathrm{~s}$ & $\begin{array}{l}1.86 \mathrm{~s}, 2.00 \mathrm{~s}(\mathrm{OAc}) \\
7.2-7.6 \mathrm{~m}(\mathrm{Tr})\end{array}$ \\
\hline $24\left({ }^{13} \mathrm{C}\right)$ & 105.52 & 40.80 & 68.28 & 69.74 & 75.06 & 62.86 & $\begin{array}{l}19.93 \\
20.60\end{array}$ & 55.00 & $\begin{array}{l}22.16,20.74(\mathrm{Me}) \\
170.48,169.55(\mathrm{C}=\mathrm{O})^{\mathrm{c})}\end{array}$ \\
\hline & & & $\begin{array}{l}1.62 \mathrm{bt} \\
\left(J_{3^{\prime}, 4}=J_{3^{\prime}, 3}=7.8\right)\end{array}$ & & & & & & \\
\hline $25\left({ }^{1} \mathrm{H}\right)$ & $4.20 \mathrm{~s}$ & & $\begin{array}{l}1.44 \mathrm{dd} \\
\left(J_{3,4}=4.4\right)\end{array}$ & $\begin{array}{c}5.00 \text { ddd } \\
\left(J_{4,5}=6.6\right)\end{array}$ & $\begin{array}{r}3.96 \text { ddd } \\
\left(J_{5,6}=4.0\right)\end{array}$ & $\begin{array}{c}3.16 \mathrm{dd}, 3.24 \mathrm{t} \\
\left(J_{6,6^{\prime}}=J_{5,6^{\prime}}=8.0\right)\end{array}$ & $\begin{array}{l}0.92 \mathrm{~s} \\
1.04 \mathrm{~s}\end{array}$ & $3.32 \mathrm{~s}$ & $\begin{array}{l}2.02 \mathrm{~s}(\mathrm{OAc}) \\
7.2-7.6 \mathrm{~m}(\mathrm{Tr})\end{array}$ \\
\hline $25\left({ }^{13} \mathrm{C}\right)$ & 99.66 & 35.34 & 43.99 & 70.28 & 71.69 & 62.28 & $\begin{array}{l}19.86 \\
21.13\end{array}$ & 57.20 & $21.33,164.62(\mathrm{OAc})^{\mathrm{d})}$ \\
\hline $\mathbf{2 6}\left({ }^{1} \mathrm{H}\right)$ & $\begin{array}{c}4.42 \mathrm{~d} \\
\left(J_{1,2}=4.0\right)\end{array}$ & $\begin{array}{c}4.56 \mathrm{dd} \\
\left(J_{2,3}=7.8\right)\end{array}$ & $4.72 \mathrm{bd}$ & & $\begin{array}{l}3.2-3.6 \mathrm{~m} \\
(2 \mathrm{H})\end{array}$ & $\begin{array}{l}3.12 \mathrm{dd} \\
\left(J_{5,6}=4.0, J_{6,6}=6.8\right)\end{array}$ & $\begin{array}{l}0.88 \mathrm{~s} \\
0.90 \mathrm{~s}\end{array}$ & $3.60 \mathrm{~s}$ & $\begin{array}{l}2.08 \mathrm{~s}, 2.12 \mathrm{~s}(\mathrm{OAc}) \\
7.2-7.6 \mathrm{~m}(\mathrm{Tr})\end{array}$ \\
\hline $26\left({ }^{13} \mathrm{C}\right)$ & 98.76 & 69.45 & 81.75 & 38.70 & 75.88 & 64.14 & $\begin{array}{l}20.67 \\
23.43\end{array}$ & 53.12 & $\begin{array}{l}24.10,24.75(\mathrm{Me}) \\
\left.167.38,168.94(\mathrm{C}=\mathrm{O})^{\mathrm{e}}\right)\end{array}$ \\
\hline $28\left({ }^{1} \mathrm{H}\right)$ & $\begin{array}{c}4.60 \mathrm{~d} \\
\left(J_{1,2}=1.2\right)\end{array}$ & $\begin{array}{c}4.22 \mathrm{bdd} \\
\left(J_{2,3}=4.0\right)\end{array}$ & $4.26 \mathrm{bd}$ & & $\begin{array}{l}3.2-3.5 \mathrm{~m} \\
(2 \mathrm{H})\end{array}$ & $\begin{array}{l}3.14 \mathrm{dd} \\
\left(J_{5,6}=2.8, J_{6,6^{\prime}}=5.4\right)\end{array}$ & $\begin{array}{l}0.82 \mathrm{~s} \\
0.86 \mathrm{~s}\end{array}$ & $3.64 \mathrm{~s}$ & $\begin{array}{l}2.02 \mathrm{bs}(6 \mathrm{H})(\mathrm{OAc}) \\
7.2-7.6 \mathrm{~m}(\mathrm{Tr})\end{array}$ \\
\hline $28\left({ }^{13} \mathrm{C}\right)$ & 100.12 & 73.16 & 78.77 & 39.02 & 77.51 & 65.98 & $\begin{array}{l}19.72 \\
22.80\end{array}$ & 55.69 & $\begin{array}{l}27.38,22.94(\mathrm{Me}) \\
166.23,178.46(\mathrm{C}=\mathrm{O})^{n}\end{array}$ \\
\hline
\end{tabular}

a) $85.85\left(\mathrm{Ph}_{3} \mathrm{C}\right), 125.87,126.65,127.77$, and $142.91(\mathrm{Ph})$. b) $87.01\left(\mathrm{Ph}_{3} \mathrm{C}\right), 127.00,127.81,128.68$, and $144.06(\mathrm{Ph}) . \quad$ c) $86.63\left(\mathrm{Ph}_{3} \mathrm{C}\right)$, 126.90, 127.72, 128.75, and $143.78(\mathrm{Ph})$. d) $85.62\left(\mathrm{Ph}_{3} \mathrm{C}\right), 126.43 .127 .53,128.23$, and $143.66(\mathrm{Ph})$. e) $81.94\left(\mathrm{Ph}_{3} \mathrm{C}\right), 127.37,127.49$, 128.68, and $144.06(\mathrm{Ph})$. f) $85.45\left(\mathrm{Ph}_{3} \mathrm{C}\right), 127.26,127.92,128.39$, and $144.14(\mathrm{Ph})$.

loromethane. The extract was washed with aqueous sodium chloride, dried with sodium sulfate, and evaporated to give crude 1,2-adduct as a syrup. The syrup was oxidized with dimethyl sulfoxide $(0.3 \mathrm{ml})$ and oxalyl dichloride $(0.2 \mathrm{ml})$ in dichloromethane $(4.5 \mathrm{ml})$ in the same manner as described for compound 14. Crude 5 was purified on a column of silica gel with hexane-ether $(3: 1)$, yield, $206 \mathrm{mg}(82 \%)$; syrup, IR $(\mathrm{NaCl}) 1700(\mathrm{C}=\mathrm{O})$ and $1660 \mathrm{~cm}^{-1}(\mathrm{C}=\mathrm{C}) .{ }^{1} \mathrm{H}$ NMR $\delta=4.96\left(1 \mathrm{H}, \mathrm{d}, J_{1,2}=3.6 \mathrm{~Hz}, \mathrm{H}-1\right), 3.44\left(1 \mathrm{H}, \mathrm{dd}, J_{2,3}=6.4 \mathrm{~Hz}\right.$, $\mathrm{H}-2$ ), 3.72 ( $1 \mathrm{H}, \mathrm{d}, \mathrm{H}-3$ ), 1.88 and 1.96 (each $3 \mathrm{H}$, each s, CMe), 3.50 ( $3 \mathrm{H}, \mathrm{s}, \mathrm{OMe})$ and 3.58 (6H, s, OMe).

Found: $\mathrm{C}, 57.66 ; \mathrm{H}, 8.17 \%$. Calcd for $\mathrm{C}_{11} \mathrm{H}_{18} \mathrm{O}_{5}$ : C, 57.38; $\mathrm{H}, \mathbf{7 . 8 8 \%}$.

Preparation of Enol Trimethylsilyl Ethers 7, 21, and 23 from Enones 6, 2, and 3. To a reaction mixture of an enone with lithium (cyano)dimethylcuprate(I) as described latter, was added after $4 \mathrm{~h}$ at $-78^{\circ} \mathrm{C}$ triethylamine $(1.7 \mathrm{ml}$ per 1.0 mmol of enone), hexamethylphosphoric triamide $(0.3 \mathrm{ml}$ per $1.0 \mathrm{mmol}$ of enone), and chlorotrimethylsilane (1.7 ml per $1.0 \mathrm{mmol}$ of enone). Being kept at $0^{\circ} \mathrm{C}$ for $1 \mathrm{~h}$, the mixture was processed in the same manner as described for 13 . The enol ethers were used for further reaction without purification.

Methyl 2,3-Dideoxy-2-C-methyl-4-O-trimethylsilyl-6-Otrityl- $\alpha$-D-threo-hex-3-enopyranoside (7). Treatment of $6^{12)}$ as described above gave $\mathbf{7}$ quantitatively, which was characterized only by ${ }^{1} \mathrm{H}$ NMR $\delta=4.72\left(1 \mathrm{H}, \mathrm{d}, J_{1,2}=4.8 \mathrm{~Hz}, \mathrm{H}-1\right)$, $2.54\left(1 \mathrm{H}, \mathrm{dq}, J_{2, \mathrm{Me}}=6.8 \mathrm{~Hz}, \mathrm{H}-2\right), 4.66(1 \mathrm{H}, \mathrm{bs}, \mathrm{H}-3), 4.22(1 \mathrm{H}$, dd, $\left.J_{5,6}=4.4 \mathrm{~Hz}, J_{6,6^{\prime}}=8.0 \mathrm{~Hz}, \mathrm{H}-5\right), 3.40\left(1 \mathrm{H}, \mathrm{t}, J_{5,6^{\prime}}=8.0 \mathrm{~Hz}\right.$, H-6), 3.52 (1H, dd, H-6'), 0.12 (9H, s, SiMe), 1.02 (3H, d, $\mathrm{C}-\mathrm{Me}), 3.62(3 \mathrm{H}, \mathrm{s}, \mathrm{OMe})$ and $7.2-7.6(15 \mathrm{H}, \mathrm{m}, \mathrm{Tr})$.

Methyl 2,3-Dideoxy-2,2-di- $C$-methyl-4- $O$-trimethylsilyl- $\alpha$ D-glycero-hex-3-enopyranoside (21). Treatment of 2 as des- cribed above gave $\mathbf{2 1}$ quantitatively, which was characterized only by ${ }^{1} \mathrm{H}$ NMR $\delta=4.62(1 \mathrm{H}, \mathrm{s}, \mathrm{H}-1), 4.68(\mathrm{lH}, \mathrm{bs}, \mathrm{H}-3)$, $3.0-3.4\left(2 \mathrm{H}, \mathrm{m}, \mathrm{H}-5\right.$ and $\left.\mathrm{H}-6^{\prime}\right), 3.84\left(1 \mathrm{H}, \mathrm{dd}, J_{5,6}=4.0 \mathrm{~Hz}\right.$, $\left.J_{6,6^{\prime}}=6.6 \mathrm{~Hz}, \mathrm{H}-6\right), 0.18(9 \mathrm{H}, \mathrm{s}, \mathrm{SiMe}), 0.98$ and 1.00 (each $3 \mathrm{H}$, each s, C-Me), $3.56(3 \mathrm{H}, \mathrm{s}, \mathrm{OMe})$, and $7.2-7.6(15 \mathrm{H}, \mathrm{m}, \mathrm{Tr})$.

Methyl 3,4-Dideoxy-4,4-di-C-methyl-2-O-trimethylsilyl-6$O$-trityl- $\alpha$-D-glycero-hex-2-enopyranoside (23). Treatment of $3^{16)}$ as described above gave 23 quantitatively, which was characterized only by ${ }^{1} \mathrm{H}$ NMR $\delta=4.70(1 \mathrm{H}, \mathrm{s}, \mathrm{H}-1), 4.94(1 \mathrm{H}$, s, H-3), $3.92\left(1 \mathrm{H}\right.$, dd, $\left.J_{5,6}=3.8 \mathrm{~Hz}, J_{5,6^{\prime}}=6.2 \mathrm{~Hz}, \mathrm{H}-5\right), 3.36$ $\left(1 \mathrm{H}, \mathrm{dd}, J_{6,6^{\prime}}=7.0 \mathrm{~Hz}, \mathrm{H}-6\right), 3.28\left(\mathrm{lH}, \mathrm{dd}, \mathrm{H}-6^{\prime}\right), 0.18(9 \mathrm{H}, \mathrm{s}$, SiMe), 0.76 and 0.84 (each $3 \mathrm{H}$, each s, C-Me), 3.66 ( $3 \mathrm{H}, \mathrm{s}$, $\mathrm{OMe})$ and $7.2-7.6(15 \mathrm{H}, \mathrm{m}, \mathrm{Tr})$.

Methyl 6-O-Benzyl-2,3-di-O-methyl-4- $C$-methyl- $\alpha$-D-galactopyranoside (13). Swern oxidation of $12,{ }^{20}$ as described for 14, gave the corresponding 4 -ulose in $84 \%$ yield. To a solution of methylmagnesium iodide $(2.17 \mathrm{mmol})$ in ether $(5$ $\mathrm{ml}$ ) was added the ulose $(227 \mathrm{mg}, 0.72 \mathrm{mmol})$ at $0{ }^{\circ} \mathrm{C}$. After $12 \mathrm{~h}$ at the same temperature, the solution was mixed with saturated aqueous ammonium chloride and extracted with dichloromethane. The extract was washed with aqueous sodium chloride, dried over magnesium sulfate, and evaporated. The crude mixture of products was fractionated on a column of silica gel with hexane-ethanol $(18: 1)$ to give 13 (187 $\mathrm{mg}, 81 \%$ ) and its 4-epimer (23 mg, 10\%).

13: Syrup, $[\alpha]_{\mathrm{D}}^{20}+62.5^{\circ}\left(c 0.86, \mathrm{CHCl}_{3}\right) ;{ }^{1} \mathrm{H}$ NMR $\delta=4.96$ $\left(1 \mathrm{H}, \mathrm{d}, J_{1,2}=3.6 \mathrm{~Hz}, \mathrm{H}-1\right), 3.24(1 \mathrm{H}, \mathrm{dd}, \mathrm{H}-2), 3.6-3.8(4 \mathrm{H}, \mathrm{m}$, $\mathrm{H}-3, \mathrm{H}-5, \mathrm{H}-6$ and $\mathrm{H}-6^{\prime}$ ), 1.06 (3H, s, C-Me), 3.44, 3.52, and 3.60 (each $3 \mathrm{H}$, each s, OMe), $4.64\left(2 \mathrm{H}\right.$, bs, $\left.\mathrm{CH}_{2} \mathrm{C}_{6} \mathrm{H}_{5}\right)$ and 7.44 $\left(5 \mathrm{H}, \mathrm{bs}, \mathrm{C}_{6} \mathrm{H}_{5}\right)$.

Found: $\mathrm{C}, 63.08 ; \mathrm{H}, 7.56 \%$. Calcd for $\mathrm{C}_{17} \mathrm{H}_{24} \mathrm{O}_{6}$ : C, 62.95; H, $7.46 \%$.

4-Epimer of 13: Syrup $[\alpha]_{D}^{20}+43.1^{\circ}$ (c $0.81, \mathrm{CHCl}_{3}$ ); 
${ }^{1} \mathrm{H}$ NMR $\delta=5.02\left(1 \mathrm{H}, \mathrm{d}, J_{1,2}=3.6 \mathrm{~Hz}, \mathrm{H}-1\right), 3.26(1 \mathrm{H}, \mathrm{dd}$, $\left.J_{2,3}=6.6 \mathrm{~Hz}, \mathrm{H}-2\right), 3.6-3.8\left(4 \mathrm{H}, \mathrm{m}, \mathrm{H}-3, \mathrm{H}-5, \mathrm{H}-6\right.$, and $\left.\mathrm{H}-6^{\prime}\right)$, $1.32(3 \mathrm{H}, \mathrm{s}, \mathrm{C}-\mathrm{Me}), 3.68(6 \mathrm{H}, \mathrm{s}, \mathrm{OMe}), 4.62\left(2 \mathrm{H}, \mathrm{bs}, \mathrm{CH}_{2} \mathrm{C}_{6} \mathrm{H}_{5}\right)$ and $7.44\left(5 \mathrm{H}, \mathrm{bs}, \mathrm{C}_{6} \mathrm{H}_{5}\right)$.

Found: $\mathrm{C}, 62.66 ; \mathrm{H}, 7.28 \%$. Calcd for $\mathrm{C}_{17} \mathrm{H}_{24} \mathrm{O}_{6}$ : C, 62.95\%; $\mathrm{H}, 7.46 \%$.

When this reaction was performed at $-78^{\circ} \mathrm{C}$, only 13 was obtained in $90 \%$ yield. Furthermore, treatment of the ulose with methyllithium in ether at $-78^{\circ} \mathrm{C}$ for $5 \mathrm{~h}$ and at room temperature overnight, gave 13 and its 4 -epimer 42 and $36 \%$ yields, respectively.

Methyl 2,3,4-Tri- $O$-methyl-4- $C$-methyl- $\alpha$-D-galacto-hexodialdo-pyranoside-(1,5) (14). Treatment of 13 with sodium hydride in $N, N$-dimethylformamide for $4 \mathrm{~h}$ and then with methyl iodide at $0^{\circ} \mathrm{C}$, gave the $4-O$-methyl derivative in $94 \%$ yield, which was hydrogenolyzed under $3 \mathrm{~atm}(1 \mathrm{~atm}=1.01 \times$ $10^{5} \mathrm{~Pa}$ ) in the presence of $10 \%$ palladium on carbon in methanol and water $(10: 1)$ at room temperature overnight to give the $O$-debenzylated derivative in $93 \%$ yield.

To a solution of oxalyl dichloride $(0.92 \mathrm{ml}, 10.8 \mathrm{mmol})$ in dichloromethane $(30 \mathrm{ml})$ was added a solution of dimethyl sulfoxide $(0.94 \mathrm{ml}, 12.9 \mathrm{mmol})$ in dichloromethane $(10 \mathrm{ml})$ at $-78^{\circ} \mathrm{C}$ and then the $O$-debenzylated derivative $(2.1 \mathrm{~g}, 8.5$ $\mathrm{mmol}$ ) at $-78^{\circ} \mathrm{C}$. The reaction mixture was kept at $-78^{\circ} \mathrm{C}$ for $30 \mathrm{~min}$, quenched with triethylamine, poured into water, and extracted with chloroform. The extract was washed with water, dried over magnesium sulfate, and evaporated to give $14(1.87 \mathrm{~g}, 90 \%)$ as colorless syrup, $[\alpha]_{\mathrm{D}}^{20}+41.6^{\circ}(c 1.30$, $\left.\mathrm{CHCl}_{3}\right) ;{ }^{1} \mathrm{H}$ NMR $\delta=5.02\left(1 \mathrm{H}, \mathrm{d}, J_{1,2}=3.0 \mathrm{~Hz}, \mathrm{H}-\mathrm{l}\right), 3.28(1 \mathrm{H}$, dd, $\left.J_{2,3}=8.0 \mathrm{~Hz}, \mathrm{H}-2\right), 3.62(1 \mathrm{H}, \mathrm{d}, \mathrm{H}-3), 3.88(1 \mathrm{H}, \mathrm{bs}, \mathrm{H}-5)$, 9.62 (1H, bs, H-6), $1.40(3 \mathrm{H}, \mathrm{s}, \mathrm{C}-\mathrm{Me})$ and $3.38,3.42,3.48$, and 3.54 (each $3 \mathrm{H}$, each s, OMe).

Found: $\mathrm{C}, 53.45 ; \mathrm{H}, 8.27 \%$. Calcd for $\mathrm{C}_{11} \mathrm{H}_{20} \mathrm{O}_{6}$ : C, 53.22; $\mathrm{H}, 8.12 \%$.

Reaction of Enones 2-5 with Lithium Dimethylcuprate(I). To a suspension of copper(I) iodide $(0.68 \mathrm{~g}, 3.6 \mathrm{mmol})$ in ether $(10 \mathrm{ml})$ was added methyllithium $(6 \mathrm{ml}$ of $1.2 \mathrm{M}$ ethereal solution, $7.2 \mathrm{mmol})$ at $0^{\circ} \mathrm{C}$ under an atmosphere of argon, and then a solution of an enone $(1.2 \mathrm{mmol})$ in ether (5 $\mathrm{ml}$ ). The reaction mixture was kept at $0^{\circ} \mathrm{C}$ for $4 \mathrm{~h}$ and processed in the same manner as described for $\mathbf{1 3}$ to give a mixture of products, which was fractionated on a column of silica gel with the solvent system given below in individual cases.

Reaction of Enones 2-5 with Lithium (Cyano)dimethylcuprate(I). To a suspension of copper(I) cyanide (424 mg, $4.73 \mathrm{mmol})$ in ether $(13 \mathrm{ml})$, was added methyllithium (12.9 $\mathrm{ml}$ of $1.2 \mathrm{M}$ ethereal solution, $9.5 \mathrm{mmol}$ ) at $-78^{\circ} \mathrm{C}$. When the solution became clear, a solution of an enone ( 1.58 $\mathrm{mmol})$ in ether $(6.5 \mathrm{ml})$ was added, and the same temperature was kept for $4 \mathrm{~h}$. The reaction mixture was processed in the same manner as described above and the results are summarized in Table 1 .

A Mixture 15 of Methyl 4,7-Dideoxy-2,3-di- $O$-methyl-4- $C$ methyl- $\alpha$-D-xylo-hept-4-enopyranoside and Its 6-Epimer. The reactions of $\mathbf{4}$ as described above gave a mixture of unseparable two epimers 15 and methyl 4-deoxy-2,3-di- $O$ methyl-4- $C$-methyl- $\beta$-L-threo-hex-4-enopyranoside (17), which were separated on a silica gel column with hexaneethyl acetate $(3: 1)$. The epimeric mixture 15 was only characterized by ${ }^{1} \mathrm{H}$ NMR.

15: Syrup, ${ }^{1} \mathrm{H}$ NMR (major): $\delta=4.80\left(\mathrm{~d}, J_{1,2}=2.0 \mathrm{~Hz}, \mathrm{H}-1\right.$ ), $3.46(\mathrm{H}-2), 3.62\left(\mathrm{bd}, J_{2,3}=6.6 \mathrm{~Hz}, \mathrm{H}-3\right), 4.54\left(\mathrm{bq}, J_{6,7}=6.4 \mathrm{~Hz}\right.$,
H-6), 1.36 (d, H-7), 1.58 (bs, C-Me) and 3.46 (s, OMe), (minor): $\delta=4.76\left(\mathrm{~d}, J_{1,2}=2.0 \mathrm{~Hz}, \mathrm{H}-1\right), 1.34(\mathrm{~d}, \mathrm{H}-7)$ and 3.38 (s, OMe).

Furthermore, conventional acetylation of $\mathbf{1 5}$ with acetic anhydride and pyridine gave $\mathbf{1 6}$ as colorless syrup, 16: ${ }^{1} \mathrm{H} N M R$ (major) $\delta=4.96$ (d, H-1), $3.56(\mathrm{H}-2), 3.74$ (d, $J_{2,3}=8.8 \mathrm{~Hz}, \mathrm{H}-3$ ), 5.76 (q, H-6), 1.74 (bs, C-Me), 1.42 (d, $\mathrm{H}-7$ ), 2.08 (s, OAc) and 3.56 (bs, OMe), (minor) $\delta=4.94$ (d, $\left.J_{1,2}=2.2 \mathrm{~Hz}, \mathrm{H}-1\right), 3.78$ (d, H-3), $5.76\left(\mathrm{q}, J_{6,7}=6.2 \mathrm{~Hz}, \mathrm{H}-6\right)$, $1.40(\mathrm{~d}, \mathrm{H}-7)$ and $3.50(\mathrm{~s}, \mathrm{OMe})$.

17: Syrup, $[\alpha]_{\mathrm{D}}^{23}+28.5^{\circ}\left(c 0.43, \mathrm{CHCl}_{3}\right)$, IR $(\mathrm{NaCl}) 3500$ $(\mathrm{OH})$ and $1610 \mathrm{~cm}^{-1}(\mathrm{C}=\mathrm{C}) ;{ }^{1} \mathrm{H}$ NMR $\delta=4.76\left(1 \mathrm{H}, \mathrm{d}, J_{1,2}=3.6\right.$ $\mathrm{Hz}, \mathrm{H}-1$ ), 3.22 (1H, dd, $\left.J_{2,3}=5.8 \mathrm{~Hz}, \mathrm{H}-2\right), 3.68(1 \mathrm{H}, \mathrm{d}, \mathrm{H}-3)$, 3.38 (2H, bs, H-6), $1.74(3 \mathrm{H}, \mathrm{bs}, \mathrm{C}-\mathrm{Me})$ and $3.42,3.44$, and 3.50 (each $3 \mathrm{H}$, each s, OMe).

Found: C, 54.88; $\mathrm{H}, 8.27 \%$. Calcd for $\mathrm{C}_{10} \mathrm{H}_{18} \mathrm{O}_{5}$ : C, 55.03; $\mathrm{H}, 8.31 \%$.

Methyl 2,3-Dideoxy-2,2-di- $C$-methyl-6- $O$-trityl- $\alpha$-D-glycerohexopyranosid-4-ulose (18). The reactions of 2 with lithium methylcuprates(I) as described above gave a mixture of $\mathbf{1 8}$ and its 5-epimer, methyl 2,3-dideoxy-2,2-di- $\mathrm{C}$-methyl-6- $\mathrm{O}$ trityl- $\beta$-L-glycero-hexopyranosid-4-ulose, which were separated on a column of silica gel with hexane-ethyl acetate $(15: 1)$.

18: Syrup, $[\boldsymbol{\alpha}]_{\mathrm{D}}^{23}+104.1^{\circ}\left(c 2.2, \mathrm{CHCl}_{3}\right)$; IR $(\mathrm{NaCl}) 1740$ $\mathrm{cm}^{-1}(\mathrm{C}=\mathrm{O})$. The ${ }^{1} \mathrm{H}$ and ${ }^{13} \mathrm{C}$ NMR data are given in Table 3 . Found: C, 78.25; $\mathrm{H}, 7.09 \%$. Calcd for $\mathrm{C}_{28} \mathrm{H}_{30} \mathrm{O}_{4}$ : C, 78.11; $\mathrm{H}, 7.02 \%$.

5-Epimer of 18: Syrup, $[\alpha]_{\mathrm{D}}^{23}+38.6^{\circ}\left(c 1.41, \mathrm{CHCl}_{3}\right)$; IR $(\mathrm{NaCl}) 1740 \mathrm{~cm}^{-1}(\mathrm{C}=\mathrm{O}),{ }^{1} \mathrm{H}$ NMR $\delta=4.36(\mathrm{lH}, \mathrm{s}, \mathrm{H}-1), 2.12$, and 2.48 (each $1 \mathrm{H}$, each $\mathrm{d}, J=16.0 \mathrm{~Hz}, \mathrm{H}-3$ and $\mathrm{H}-3^{\prime}$ ), 4.12 $\left(1 \mathrm{H}, \mathrm{dd}, J_{5,6}=3.6 \mathrm{~Hz}, J_{5,6}=5.4 \mathrm{~Hz}, \mathrm{H}-5\right), 3.4-3.6(2 \mathrm{H}, \mathrm{m}$, $\mathrm{H}-6), 1.02$ and 1.00 (each $3 \mathrm{H}$, each s, $\mathrm{C}_{2}-\mathrm{Me}$ ), $3.38(3 \mathrm{H}, \mathrm{s}$, $\mathrm{OMe})$ and $7.1-7.5(\mathrm{~m}, \mathrm{Tr})$.

Found: $\mathrm{C}, 78.05 ; \mathrm{H}, 7.02 \%$. Calcd for $\mathrm{C}_{28} \mathrm{H}_{30} \mathrm{O}_{4}: \mathrm{C}, 78.11 ; \mathrm{H}$, $7.02 \%$.

Methyl 3,4-Dideoxy-4,4-di- $C$-methyl-6-O-trityl- $\alpha$-D-glycerohexopyranosid-2-ulose (19). The reaction of 3 with lithium methylcuprates(I) as described above gave crude 19 , which was purified on a column of silica gel with hexane-ethyl acetate $(10: 1)$, syrup, $[\alpha]_{\mathrm{D}}^{23}+27.1^{\circ}\left(c 1.5, \mathrm{CHCl}_{3}\right), \mathrm{IR}(\mathrm{NaCl})$ $1740 \mathrm{~cm}^{-1}(\mathrm{C}=\mathrm{O})$. The ${ }^{1} \mathrm{H}$ and ${ }^{13} \mathrm{CNMR}$ data are given in Table 3.

Found: C, 77.93; $\mathrm{H}, 7.24 \%$. Calcd for $\mathrm{C}_{28} \mathrm{H}_{30} \mathrm{O}_{4}: \mathrm{C}, 78.11 ; \mathrm{H}$, $7.02 \%$.

Methyl 4,7-Dideoxy-2,3-di- $O$-methyl-4,4-di- $C$-methyl- $\alpha$-Dxylo-heptopyranosid-6-ulose (20). The reactions of 5 with lithium methylcuprates(I) as described above gave crude $\mathbf{2 0}$, which was purified on a column of silica gel with hexaneethyl acetate $(4: 1)$, syrup, $[\alpha]_{\mathrm{D}}^{23}+55.2^{\circ}$ (c $0.44, \mathrm{CHCl}_{3}$ ), ${ }^{1} \mathrm{H}$ NMR $\delta=4.68\left(1 \mathrm{H}, \mathrm{d}, J_{1,2}=3.2 \mathrm{~Hz}, \mathrm{H}-1\right), 3.4-3.6(2 \mathrm{H}, \mathrm{m}$, $\mathrm{H}-2$ ), 1.36 and 1.56 (each $3 \mathrm{H}$, each $\mathrm{s}, \mathrm{C}_{4}-\mathrm{Me}$ ), $1.82(3 \mathrm{H}, \mathrm{s}$, $\left.\mathrm{C}_{6}-\mathrm{Me}\right), 3.52(6 \mathrm{H}, \mathrm{s}, \mathrm{OMe})$ and $3.46(3 \mathrm{H}, \mathrm{s}, \mathrm{OMe})$.

Found: C, 58.29; H, 9.20\%. Calcd for $\mathrm{C}_{12} \mathrm{H}_{22} \mathrm{O}_{5}$ : C, 58.51; H, $9.00 \%$.

Hydroborations of 21 and 23. (i) With Borane in Tetrahydrofuran. To a solution of a enol trimethylsilyl ether $(503 \mathrm{mg}, 1.0 \mathrm{mmol})$ in tetrahydrofuran $(6.5 \mathrm{ml})$, was added calculated amount of borane (the amount given in Table 2, 1 $\mathrm{M}$ solution in tetrahydrofuran) at $0^{\circ} \mathrm{C}$ under an atmosphere of argon. Being kept at room temperature for $6 \mathrm{~h}$, the reaction mixture was poured into water $(10 \mathrm{ml})$. To a separated organic layer was added, at $0^{\circ} \mathrm{C}$ with vigorous stirring, $10 \%$ 
aqueous sodium hydroxide $(5 \mathrm{ml})$ and hydrogen peroxide $(30 \%, 5 \mathrm{ml})$ and the stirring was continued at $45^{\circ} \mathrm{C}$ for 30 min. The reaction mixture was extracted with chloroform and the extract was washed with aqueous sodium chloride, dried over magnesium sulfate, and evaporated to give a mixture of products as a colorless syrup. The mixture was acetylated conventionally with acetic anhydride $(2 \mathrm{ml})$ and pyridine $(3 \mathrm{ml})$. In the case of 21 , fractionation of the products on a column of silica gel with hexane-ethyl acetate $(15: 1)$ afforded 22, 24, and 25 in the yields given in Table 2, while in the case of 23 with hexane-ethyl acetate (25:1) 26, 27, and 28.

(ii) With Sodium Borohydride and Titanium(III) Chloride. To a mixture of sodium borohydride $(100 \mathrm{mg}, 2.6$ $\mathrm{mmol}$ ) and 18-crown-6 (132 mg, $0.5 \mathrm{mmol})$ in tetrahydrofuran $(10 \mathrm{ml})$ was added titanium(III) chloride $(85 \mathrm{mg}, 0.55$ $\mathrm{mmol}$ ), and the mixture was stirred at $30^{\circ} \mathrm{C}$ for $1 \mathrm{~h}$ under argon. After the color of the solution turned to be dark violet, a solution of enol silyl ether $(503 \mathrm{mg}, 1.0 \mathrm{mmol}$ ) in tetrahydrofuran $(5.5 \mathrm{ml})$ was added, and the mixture was stirred at the same temperature for $8 \mathrm{~h}$. To the mixture was added methanolic sodium methoxide ( $3 \mathrm{ml}$ of $3 \mathrm{M}$ solution) and then $30 \%$ aqueous $\mathrm{H}_{2} \mathrm{O}_{2}(5 \mathrm{ml})$. Being kept at $40^{\circ} \mathrm{C}$ for 1 $\mathrm{h}$, the reaction mixture was processed in the same manner as described above.

(iii) With Sodium Borohydride and Cobalt(II) Chloride. To a suspension of cobalt(II) chloride $(325 \mathrm{mg}, 2.5$ $\mathrm{mmol})$ in tetrahydrofuran $(8 \mathrm{ml})$, was added with stirring at $0^{\circ} \mathrm{C}$ under argon sodium borohydride $(195 \mathrm{mg}, 5 \mathrm{mmol})$. After $1 \mathrm{~h}$ a solution of enol silyl ether $(0.66 \mathrm{mmol})$ in tetrahydrofuran $(3 \mathrm{ml})$ was added after stirring at room temperature for $1.5 \mathrm{~h}$, and the mixture was stirred at room temperature for $8 \mathrm{~h}$. The successive oxidation was performed as described for hydroboration with titanium(III) chloride and sodium borohydride.

(iv) With Disiamylborane. To a $0.6 \mathrm{M}$ solution of disiamylborane $(2 \mathrm{ml})$ in tetrahydrofuran was added a solution of enol silyl ether $(503 \mathrm{mg}, 1.0 \mathrm{mmol})$ in tetrahydrofuran $(6 \mathrm{ml})$ at room temperature, and the mixture was stirred at room temperature for $8 \mathrm{~h}$. The oxidation was performed in the same manner as described above.

(v) With 9-Borabicyclo[3.3.1]nonane (9-BBN). To a $0.5 \mathrm{M}$ solution of 9-borabicyclo[3.3.1] nonane $(2.4 \mathrm{ml})$ in tetrahydrofuran was added a solution of enol silyl ether $(503 \mathrm{mg}, 1.0$ $\mathrm{mmol})$ in tetrahydrofuran $(5.5 \mathrm{ml})$. The mixture was heated under reflux for $6 \mathrm{~h}$. The oxidation was performed in the same manner as described above.

Methyl 3,4-Di- $O$-acetyl-2-deoxy-2,2-di- $C$-methyl-6- $O$-trityl$\alpha$-D-arabino-hexopyranoside (24). Hydroboration of 21 with 3 equivalents of diborane in the same manner as described above gave a mixture of 24, methyl 4-O-acetyl-2,3dideoxy-2,2-di- $C$-methyl-6- $O$-trityl- $\alpha$-D-erythro-hexopyranoside (25), and methyl 2,3,4-trideoxy-2,2-di- $C$-methyl-6-O-trityl$\alpha$-D-glycero-hex-3-enopyranoside (22).

24: Syrup, $[\alpha]_{\mathrm{D}}^{23}+150.6^{\circ}\left(c \mathrm{l} 1.2, \mathrm{CHCl}_{3}\right)$, IR (NaCl) 1720 $\mathrm{cm}^{-1}(\mathrm{C}=\mathrm{O})$. The ${ }^{1} \mathrm{H}$ and ${ }^{13} \mathrm{C}$ NMR data are given in Table 3 .

Found: C, 72.11; $\mathrm{H}, 6.85 \%$. Calcd for $\mathrm{C}_{32} \mathrm{H}_{36} \mathrm{O}_{7}$ : C, 72.16; $\mathrm{H}, 6.81 \%$.

25: Syrup, $[\alpha]_{\mathrm{D}}^{23}+13.7^{\circ}\left(c 0.97, \mathrm{CHCl}_{3}\right)$, IR $(\mathrm{NaCl}) 1720$ $\mathrm{cm}^{-1}(\mathrm{C}=\mathrm{O})$. The ${ }^{1} \mathrm{H}$ and ${ }^{13} \mathrm{C}$ NMR data are given in Table 3 .

Found: $\mathrm{C}, 75.68 ; \mathrm{H}, 7.50 \%$. Calcd for $\mathrm{C}_{30} \mathrm{H}_{34} \mathrm{O}_{5}$ : C, 75.92; $\mathrm{H}, 7.22 \%$.

22: Syrup, $[\alpha]_{\mathrm{D}}^{23}+12.0^{\circ}\left(c \mathrm{1} .14, \mathrm{CHCl}_{3}\right)$, IR $(\mathrm{NaCl}) 1680$
$\mathrm{cm}^{-1}(\mathrm{C}=\mathrm{C}),{ }^{1} \mathrm{H}$ NMR $\delta=4.44(1 \mathrm{H}, \mathrm{s}, \mathrm{H}-1), 5.62(1 \mathrm{H}, \mathrm{bd}$ $\left.J_{3,4}=9.2 \mathrm{~Hz}, \mathrm{H}-3\right), 5.58\left(1 \mathrm{H}\right.$, bdd, $\left.J_{4,5}=4.6 \mathrm{~Hz}, \mathrm{H}-4\right), 3.82(1 \mathrm{H}$, bdd, $\left.J_{5,6}=4.0 \mathrm{~Hz}, J_{5,6},=6.8 \mathrm{~Hz}, \mathrm{H}-5\right), 3.12(2 \mathrm{H}, \mathrm{m}, \mathrm{H}-6), 1.02$ and 1.10 (each $3 \mathrm{H}$, each $\left.\mathrm{s}, \mathrm{C}_{2}-\mathrm{Me}\right), 3.52(3 \mathrm{H}, \mathrm{s}, \mathrm{OMe})$ and 7.2-7.6 (15H, m, Tr).

Found: C, 80.96; $\mathrm{H}, 7.43 \%$. Calcd for $\mathrm{C}_{28} \mathrm{H}_{30} \mathrm{O}_{3}$ : C, 81.13; $\mathrm{H}, \mathbf{7 . 2 9 \%}$.

Methyl 2,3-Di- $O$-acetyl-4-deoxy-4,4-di- $C$-methyl-6-O-trityl$\alpha$-D-xylo-hexopyranoside (26) and Methyl 2,3-Di-O-acetyl-4deoxy-4,4-di- $C$-methyl-6- $O$-trityl- $\alpha$-D-arabino-hexpyranoside (28). Hydroboration of $\mathbf{2 3}$ in the same manner as described above gave 26, 28, and methyl 2-O-acetyl-3,4-dideoxy-4,4-di$C$-methyl-6- $O$-trityl- $\alpha$-D-erythro-hexopyranoside (27).

26: Syrup, $[\alpha]_{\mathrm{D}}^{23}+91.5^{\circ}\left(c \mathrm{c} 0.89, \mathrm{CHCl}_{3}\right)$, IR $(\mathrm{NaCl}) 1700$ and $1710 \mathrm{~cm}^{-1}(\mathrm{C}=\mathrm{O})$. The ${ }^{1} \mathrm{H}$ and ${ }^{13} \mathrm{CNMR}$ data are given in Table 3.

Found: C, 72.02; $\mathrm{H}, 6.92 \%$. Calcd for $\mathrm{C}_{32} \mathrm{H}_{36} \mathrm{O}_{7}: \mathrm{C}, 72.16$; $\mathrm{H}, 6.81 \%$.

28: Syrup, $[\alpha]_{\mathrm{D}}^{23}+113.1^{\circ}$ (c $1.3, \mathrm{CHCl}_{3}$ ), IR (NaCl) 1720 $\mathrm{cm}^{-1}(\mathrm{C}=\mathrm{O})$. The ${ }^{1} \mathrm{H}$ and ${ }^{13} \mathrm{C}$ NMR data are given in Table 3 .

Found: $\mathrm{C}, 71.96 ; \mathrm{H}, 6.79 \%$. Calcd for $\mathrm{C}_{32} \mathrm{H}_{36} \mathrm{O}_{7}$ : C, 72.16; H, $6.81 \%$.

27: Mp $128-130^{\circ} \mathrm{C},[\alpha]_{\mathrm{D}}^{23}+51.2^{\circ}$ (c $1.2, \mathrm{CHCl}_{3}$ ), IR ( $\mathrm{NaCl}) 1710 \mathrm{~cm}^{-1}(\mathrm{C}=\mathrm{O}),{ }^{1} \mathrm{H}$ NMR $\delta=4.87\left(1 \mathrm{H}, \mathrm{dd}, J_{1,2}=3.4\right.$ $\left.\mathrm{Hz}, J_{1,3}=0.6 \mathrm{~Hz}, \mathrm{H}-1\right), 4.89\left(1 \mathrm{H}\right.$, ddd, $J_{2,3}=5.3 \mathrm{~Hz}, J_{2,3^{\prime}}=13.3$ $\mathrm{Hz}, \mathrm{H}-2$ ), 1.42 and 1.76 (each $1 \mathrm{H}$, each dd, $J_{3,3}=11.5 \mathrm{~Hz}, \mathrm{H}-3$ and $\left.\mathrm{H}-3^{\prime}\right), 3.74\left(1 \mathrm{H}\right.$, dd, $\left.J_{5,6}=3.8 \mathrm{~Hz}, J_{5,6^{\prime}}=9.8 \mathrm{~Hz}, \mathrm{H}-5\right), 3.10$ $\left(1 \mathrm{H}, \mathrm{dd}, J_{6,6^{\prime}}=9.8 \mathrm{~Hz}, \mathrm{H}-6\right), 3.22\left(1 \mathrm{H}, \mathrm{t}, \mathrm{H}-6^{\prime}\right), 0.78$ and 0.82 (each $3 \mathrm{H}$, each s, C-Me), $2.16(3 \mathrm{H}, \mathrm{s}, \mathrm{OAc}), 3.58(3 \mathrm{H}, \mathrm{s}, \mathrm{OMe})$ and 7.2-7.6 (m, Tr). The data for H-1, H-2, H-3, and H-3', were obtained by simulation.

Found: $\mathrm{C}, 75.63 ; \mathrm{H}, 6.95 \%$. Calcd for $\mathrm{C}_{30} \mathrm{H}_{34} \mathrm{O}_{5}$ : C, 75.92; $\mathrm{H}, 7.22 \%$.

${ }^{1} \mathrm{H}$ NMR data of deacetylated 27: $\delta=4.86\left(1 \mathrm{H}, \mathrm{d}, J_{1,2}=3.8\right.$ $\mathrm{Hz}, \mathrm{H}-1), 3.58\left(1 \mathrm{H}, \mathrm{dt}, J_{2,3}=3.8 \mathrm{~Hz}, J_{2,3}=6.2 \mathrm{~Hz}, \mathrm{H}-2\right), 1.44$ and 1.64 (each $1 \mathrm{H}$, each dd, $J_{3,3}=12.0 \mathrm{~Hz}, \mathrm{H}-3$ and $\mathrm{H}-3^{\prime}$ ), 3.59 $\left(1 \mathrm{H}, \mathrm{dd}, J_{5,6}=4.0 \mathrm{~Hz}, J_{5,6}=7.6 \mathrm{~Hz}, \mathrm{H}-5\right), 3.14(1 \mathrm{H}$, dd, $J_{6,6^{\prime}}=7.6 \mathrm{~Hz}, \mathrm{H}-6$ ), 3.28 ( $1 \mathrm{H}, \mathrm{t}, \mathrm{H}-6^{\prime}$ ), 0.72 and 0.80 (each $3 \mathrm{H}$, each s, C-Me), $3.60(3 \mathrm{H}, \mathrm{s}, \mathrm{OMe})$ and $7.2-7.6(\mathrm{~m}, \mathrm{Tr})$.

\section{References}

1) S. Hanessian, "Total Synthesis of Natural Products: The 'Chiron' Approach," Pergamon Press, Oxford (1983).

2) Y. Ali and W. A. Szarek, Carbohydr. Res., 67, Cl7 (1978).

3) Y. Chapleur, J. Chem. Soc., Chem. Commun., 1983, 141 .

4) B. Fraser-Reid, H. Tsang, D. B. Tulshian, and K. M. Sun, J. Org. Chem., 46, 3764 (1981).

5) S. Nagarajan and K. L. Rinehardt, Jr., J. Org. Chem., 50, 380 (1985)

6) H. Hashimoto, N. Kawauchi, and J. Yoshimura, Chem Lett., 1985, 965.

7) E. A. Jeffery, A. Meisters, and T. Mole, Aust. J. Chem., 27, 2569 (1974).

8) M. T. Reetz J. Westermann, and R. Steinbach, J, Chem. Soc., Chem. Commun., 1981, 237.

9) G. H. Posner and D. J. Brunelle, J. Org. Chem., 38, 2747 (1973).

10) a) W. Oppolzer and T. Godel, J. Am. Chem. Soc., 100, 2583 (1978); b) K. Yamada, Y. Kyotani, S. Manabe, and H. Suzuki, Tetrahedron, 35, 293 (1979). 
11) G. Bianchetti, A. Maccioni, and M. Secci, Ann. Chem. (Rome), 60, 483 (1970).

12) B. Fraser-Reid, A. McLean, E. W. Usherwood, M. Yunker, Can. J. Chem., 48, 2877 (1970).

13) Y. Ito, T. Hirano, and T. Saegusa, J. Org. Chem., 43, 1011 (1978)

14) J. Tsuji, I. Minami, I. Shimizu, and H. Kataoka, Chem. Lett., 1984, 1133.

15) D. R. Hicks and B. Fraser-Reid, Can. J. Chem., 53, 2017 (1975).

16) B. J. Fizsimmons, D. E. Plaumann, B. Fraser-Reid, Tetrahedron Lett., 1979, 3925.

17) T. Imamoto, T. Kusumoto, Y. Tawarayama, T. Sugiura, T. Mita, Y. Hatanaka, and M. Yokoyama, J. Org. Chem., 49, 3904 (1984).

18) These addition reactions are described in the succeeding paper: N. Kawauchi and H. Hashimoto, Bull. Chem. Soc. Jpn (In contribution).

19) M. N. Bhattacharjea, M. K. Chaudhuri, H. S. Dasgu- pat, N. Roi, and D.T. Khathing, Synthesis, 1982, 588.

20) D. J. Garegg and H. Hultberg, Carbohydr. Res., 108, 97 (1982).

21) S. Hanessian, G. Rancourt, and Y. Guindon, Can. J. Chem., 56, 1843 (1978).

22) W. Carruthers, "Compounds of Zinc, Cadmium and Mercury, and of Copper, Silver and Gold in Organic Synthesis" in "Comprehensive Organometallic Chemistry," ed by G. Wilkinson, F. G. A. Stone, and E. W. Abel, Pergamon Press (1982), Vol 7, p. 685.

23) a) G. L. Larson, D. Hernandex, and A. Hernandex, J. Organomet. Chem., 76, 9 (1974); b) J.-P. Lepoittevin and C. Benezra, Tetrahedron Lett., 25, 2505 (1984).

24) H. S. Lee, K. Isagawa, H. Toyoda, and Y. Otsuji, Chem. Lett., 1984, 673.

25) N. Satyanarayana and M. Periasamy, Tetrahedron Lett., 25, 2501 (1984).

26) G. L. Larson and A. Hernandex, J. Organomet. Chem., 102, 123 (1975). 